\title{
NISSOLIA RUDDIAE (LEGUMINOSAE, PAPILIONOIDEAE), UNA ESPECIE NUEVA DE LA CUENCA DEL BALSAS, MÉXICO
}

\author{
Ramiro Cruz Durán ${ }^{1}$ y Mario Sousa S. ${ }^{2}$ \\ ${ }^{1}$ Herbario de la Facultad de Ciencias, UNAM, Apdo. postal 70-399 \\ 04510 México, D.F. \\ ${ }^{2}$ Herbario Nacional, Instituto de Biología, UNAM, Apdo. postal 70-367 \\ 04510 México, D.F.
}

\section{RESUMEN}

Se describe e ilustra Nissolia ruddiae (Leguminosae, Papilionoideae), una especie nueva de la Cuenca del Balsas en los estados de Guerrero, Morelos y Puebla, México. Esta planta es afin a $N$. hintonii Sandwith, pero difiere por sus inflorescencias fasciculadas, pubescencia glandular-setácea escasa, hojas más grandes y partes florales más pequeñas, de diferente forma.

Palabras clave: cuenca del Balsas, Guerrero, Leguminosae, México, Morelos, Nissolia, Puebla.

\section{ABSTRACT}

Nissolia ruddiae (Leguminosae, Papilionoideae), a new species from the Balsas river basin in the states of Guerrero, Morelos and Puebla, Mexico, is described and illustrated. It is similar to $N$. hintonii Sandwith, but differs in possessing fasciculate inflorescences, pubescence of scattered glandular setae, larger leaves, and smaller floral parts, different in form. Puebla.

Key words: Balsas river basin, Guerrero, Leguminosae, Mexico, Morelos, Nissolia,

\section{INTRODUCCIÓN}

Según Rudd (1981), el género Nissolia (Leguminosae, Papilionoideae) se distribuye en los trópicos americanos, pero mayormente en México. El género pertenece a la subtribu Ormocarpinae Rudd de la tribu Aeschynomeneae (Benth.) 
Hutch. Esta subtribu se caracteriza por presentar hojas con folíolos imparipinnados, flores pediceladas, hipantio campanulado, lóbulos del cáliz subiguales, ovario estipitado y fruto con marcada venación longitudinal.

Hasta el momento el género involucra 14 especies y una variedad, de las que todas habitan en México, excepto Nissolia fruticosa Jacq. var. guatemalensis (Rose) Rudd.

Al examinar ejemplares del género Nissolia para el estado de Guerrero se detectaron plantas similares a Nissolia hintonii Sandwith (Cuadro 1), pero que difieren de ésta en varias características; entre las principales, el tipo de inflorescencia, así como el tamaño y forma de las partes florales. En consecuencia se propone como una especie nueva a:

Nissolia ruddiae R. Cruz \& M. Sousa, sp. nov. Fig. 1.

Nissoliae hintonii affinis sed cum setis glandulosis sparsis; foliis (10.5-)11.5$15(-16.5) \mathrm{cm}$ longis; inflorescentiis fasciculatis cum (1-)2-8(-12) floribus; flore 9-11 mm longo, pedunculo 1-3(-4) mm longo; bractea inter pedunculum et pedicellum 4-5 mm longa, 1-1.5 mm lata; vexillo ovato, 10-11 mm longo, 7-7.5 mm lato; androecio 7$7.5 \mathrm{~mm}$ longo; pistillo 7-8 mm longo; stylo 3.5-4 mm longo; fructu cum articulo fertili (0.9-)1.2-1.5 cm longo, (0.6-)0.7 cm lato; articulo sterili (1.9-)2.1-2.6 cm longo, 0.7$0.9(-1) \mathrm{cm}$ lato.

Bejuco semileñoso, de hasta $3 \mathrm{~m}$ de largo, tallos con escasas setas glandulares. Hojas 5-folioladas, (10.5-)11.5-15(-16.5) cm de largo y (5-)6-8(-9.3) cm de ancho (incluyendo el pecíolo); estípulas triangulares, 4-5 $\mathrm{mm}$ de largo y $2 \mathrm{~mm}$ de ancho, glandular-dentadas, glabras, con glándulas en la base por el haz; pecíolo 3.8-6(-7.5) cm de largo, con algunas setas glandulares próximas a la base; folíolos orbiculares a elípticos, membranáceos, glabros; los folíolos laterales (1.7-)1.9$3.9(-4.5) \mathrm{cm}$ de largo (no incluyendo peciólulo y mucrón) y (1.7-)2-3.5 cm de ancho, opuestos a ligeramente alternos en algunas hojas, con peciólulo de 2-2.5 $\mathrm{mm}$ de largo, la base redondeada a ligeramente atenuada, el ápice obtuso, terminando en un mucrón de $2 \mathrm{~mm}$; el folíolo terminal (2.9-)3.6-5.2 cm de largo y (2.6-)2.9-3.9(-4.2) cm de ancho, con peciólulo de $2-2.5 \mathrm{~mm}$ de largo, la base redondeada a brevemente atenuada, el ápice obtuso (en ocasiones brevemente emarginado) y terminando en un mucrón de 2-2.5 mm. Inflorescencias axilares, fasciculadas con (1-)2-8(-12) flores; pedúnculos 1-3(-4) $\mathrm{mm}$ de largo (en algunos casos ausentes), en ocasiones con algunos pelos glandulares en la base. Flor 9-11 mm de largo; pedicelos 8-9(-10) $\mathrm{mm}$ de largo, con setas glandulares, la bráctea en la base 4-5 mm de largo y 1-1.5 mm de ancho, glandular-dentada en el margen, con glándulas en la unión con el pedicelo; cáliz 6-8 mm de largo (incluyendo lóbulos), el tubo 3-3.5 $\mathrm{mm}$ de largo, con setas 
Cuadro 1. Diferencias morfológicas entre $N$. hintonii Sandwith y $N$. ruddiae sp. nov. Las características de $N$. hintonii fueron transcritas en parte de Rudd (1956).

\begin{tabular}{|c|c|c|}
\hline Características & N. hintonii Sandwith & N. ruddiae sp. nov. \\
\hline Pubescencia & $\begin{array}{l}\text { setas glandulares } \\
\text { abundantes }\end{array}$ & $\begin{array}{l}\text { setas glandulares } \\
\text { escasas }\end{array}$ \\
\hline Tamaño de estípulas (mm) & $5-9 \times 1.5-2$ & $4-5 \times 2$ \\
\hline Base de la estípula & con glándulas escasas & con glándulas abundantes \\
\hline Largo de hojas (cm) & $4-8$ & $(10.5-) 11.5-15(-16.5)$ \\
\hline Inflorescencia & $\begin{array}{l}\text { racemosa-paniculada, con } \\
\text { más de } 12 \text { flores }\end{array}$ & $\begin{array}{l}\text { fasciculada con } \\
(1-) 2-8(12) \text { flores }\end{array}$ \\
\hline Largo de la flor (mm) & $12-15$ & $9-11$ \\
\hline Largo del cáliz (mm) & 6-7.5, ciliado & 6-8, pubescente \\
\hline Tamaño del estandarte $(\mathrm{mm})$ & $12-13 \times 11$ & $10-11 \times 7-7.5$ \\
\hline Forma del estandarte & orbicular & ovado \\
\hline Largo del androceo (mm) & $11-12$ & $7-7.5$ \\
\hline Largo del tubo estaminal (mm) & $5-7$ & $4-5$ \\
\hline $\begin{array}{l}\text { Largo de los filamentos, } \\
\text { parte libre }(\mathrm{mm})\end{array}$ & $\begin{array}{l}\text { similares en longitud, } \\
3-6\end{array}$ & $\begin{array}{l}\text { desiguales en longitud, } \\
(0.5-) 1 \text { y } 2\end{array}$ \\
\hline Largo del pistilo (mm) & $12-13$ & $7-8$ \\
\hline Largo del estilo (mm) & $6-9$ & $3.5-4$ \\
\hline Artículo fértil (fruto) $(\mathrm{cm})$ & $0.5-1 \times 0.4-0.8$ & $1.2-1.5 \times 0.7$ \\
\hline Artículo estéril (cm) & $1.5-2(-2.5) \times 1-2$ & $2.1-2.6 \times 0.7-0.9$ \\
\hline Semillas (mm) & $6 \times 4 \times 1.5-2$ & $5 \times 3 \times 1$ \\
\hline Distribución conocida & $\begin{array}{l}\text { Edo. de México, Guerrero } \\
\text { (parte norte; mpio. Taxco) }\end{array}$ & $\begin{array}{l}\text { Guerrero (parte este- } \\
\text { noreste), Puebla, Morelos }\end{array}$ \\
\hline Altitud (m) & (1340-) 1600-2030 & $650-1420$ \\
\hline Vegetación & $\begin{array}{l}\text { bosque de encino, transi- } \\
\text { ción bosque de encino } \\
\text { bosque tropical caducifolio }\end{array}$ & $\begin{array}{l}\text { bosque tropical caducifolio } \\
\text { (selva baja caducifolia) }\end{array}$ \\
\hline
\end{tabular}




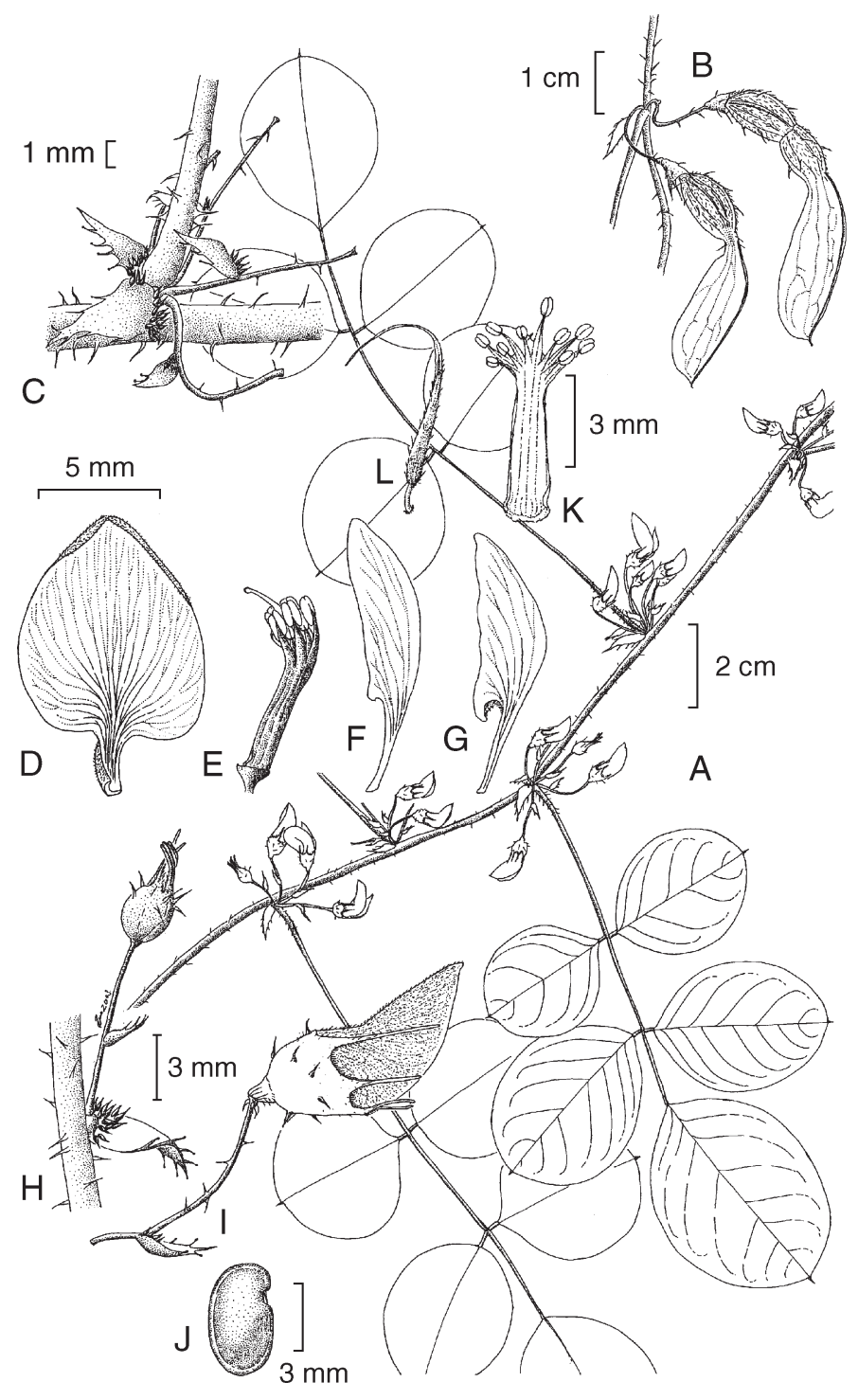

Fig. 1. Nissolia ruddiae sp. nov. A. rama con flores; B. frutos maduros; C. fascículo floral, pedúnculos, brácteas y pedicelos, también son apreciables las estípulas; D. estandarte; E. columna estaminal; F. ala; G. quilla; H. flor en botón; I. flor parcialmente en antesis, glándulas en la parte basal de la bráctea; J. semilla; K. columna estaminal en disección; L. pistilo. (A, D, E, F y G, J. C. Soto Núñez y E. Martínez Salas, 3970 (MEXU); B y J, J. L. Contreras 607 (FCME, MEXU); C, H e I, J. Hernández s.n. (FCME, MEXU); K y L., V. Aguilar 54 (FCME)). 
glandulares de 1-1.5 mm de largo, piloso en el margen (en algunos casos también piloso en la superficie), los lóbulos 3-4 mm de largo, pubescentes en la cara interna, los vexilares ligeramente más largos que los carinales; corola amarillo-verdosa, el estandarte 10-11 $\mathrm{mm}$ de largo y 7-7.5 $\mathrm{mm}$ de ancho, ovado, pubescente en la cara externa, ligeramente reflejo, la uña 2-3 mm de largo, las alas 11-12 $\mathrm{mm}$ de largo y $2.5 \mathrm{~mm}$ de ancho, glabras, uña $4 \mathrm{~mm}$ de largo, el lóbulo $0.5 \mathrm{~mm}$, quilla $10-11 \mathrm{~mm}$ de largo y $4.5 \mathrm{~mm}$ de ancho, glabra, la uña $6 \mathrm{~mm}$ de largo, la parte unida $5 \mathrm{~mm}$, el lóbulo $1 \mathrm{~mm}$, pubescente por el lado interno; androceo 7-7.5 $\mathrm{mm}$ de largo, el tubo estaminal 4-5 $\mathrm{mm}$ de largo y ca. $1 \mathrm{~mm}$ de ancho, partes libres de los filamentos desiguales en longitud y dispuestos de manera alterna, los cortos (0.5-)1 mm (formando un verticilo interno), y los largos $2 \mathrm{~mm}$, las anteras 0.8-1 mm de largo; pistilo 7-8 $\mathrm{mm}$ de largo, canescente-seríceo, con algunas setas glandulares amarillas, sobre estípite de ca. $1 \mathrm{~mm}$, el ovario 4-5 $\mathrm{mm}$ de largo, el estilo 3.5-4 $\mathrm{mm}$ de largo, curvado. Fruto 2.4-3.6(-5) cm de largo y $0.6-0.8 \mathrm{~cm}$ de ancho (con pedicelos 10$12(-14) \mathrm{mm}$ de largo y cáliz persistente), ligeramente curvado, el estípite $2 \mathrm{~mm}$ de largo, con 1-3 artículos canescente-seríceos, con algunas setas glandulares hacia la base, el artículo fértil (0.9-)1.2-1.5 cm de largo y (0.6-)0.7 cm de ancho, el estéril (1.9-)2.1-2.6 cm de largo y 0.7-0.9(-1) cm de ancho. Semillas $5 \mathrm{~mm}$ de largo, $3 \mathrm{~mm}$ de ancho y $1 \mathrm{~mm}$ de grosor, elípticas, cafés.

TIPO: México, Guerrero. Mpio. Iguala: Mexicaltepec, $8 \mathrm{~km}$ al NE de Iguala, selva baja caducifolia, altitud $800 \mathrm{~m}, 6$ jul. 1982 (flor y frutos jóvenes), J. C. Soto Núñez y E. Martínez Salas 3970 (Holotipo e isotipo: MEXU).

Esta especie crece en suelos someros, yesosos, arcillosos, pedregosos, en caliza de la formación Morelos y de origen calizo-arenoso rocoso. Se desarrolla en altitudes de 650 a 1420 m, en selva baja caducifolia (sensu Miranda y Hernández, 1963) o bosque tropical caducifolio (sensu Rzedowski, 1978) y asociada a especies como Acacia farnesiana (L.) Willd., Bursera longipes (Rose) Standl., B. lancifolia (Schltdl.) Engl., Neobuxbaumia mezcalaensis Bravo, Pseudosmodingium sp. y varias especies de Leguminosae. Florece de junio a agosto y fructifica de octubre a noviembre.

Material adicional examinado: México, Guerrero. Mpio. Huamuxtitlán: Huamuxtitlán, $4.5 \mathrm{~km}$ al N, selva baja caducifolia, altitud 1180 m, 23 jun. 1983 (flor), J. Hernández s.n. (FCME, MEXU); Tilapa, cerro El Caballo Moro, $2 \mathrm{~km}$ al S de Huamuxtitlán, bosque tropical caducifolio, altitud 850 m, 18 oct. 1987 (fruto), J. L. Contreras Jiménez 2204 (FCME); Huamuxtitlán, $6 \mathrm{~km}$ al SE, selva baja caducifolia, altitud 900 m, 24 jun. 1981 (flor), V. Aguilar 54 (FCME, MEXU). Mpio. Xochihuehuetlán: paraje La Huazarca, $2.5 \mathrm{~km}$ al N de Jilotepec, cerro Xilotzin, bosque 
tropical caducifolio, altitud 1420 m, 1758'19" N 98³0'03" O, 28 jul. 1993 (flor), E. Moreno Gutiérrez et al. 319 (FCME). Mpio. Eduardo Neri: Xochipala, $2 \mathrm{~km}$ al $\mathrm{S}$, selva caducifolia, altitud $1070 \mathrm{~m}, 1$ jul. 1989 (flor en botón), M. Sousa S., J. C. Soto Núñez y C. Catalán 13225 (MEXU); cañada aproximadamente $0.2 \mathrm{~km}$ al E de Venta Vieja, km 64, carretera Iguala-Chilpancingo, bosque tropical caducifolio, 12 nov. 1980 (fruto), J. L. Contreras Jiménez 607 (FCME, MEXU); Cañón del Zopilote, desviación Filo de Caballo [desviación a Xochipala], carretera MéxicoAcapulco (Casa Verde), bosque tropical caducifolio, altitud $650 \mathrm{~m}, 10$ jul. 1981 (flor), M. Ramírez de M. 9 (FCME, MEXU). Mpio. Copalillo: Tlalcozotitlán, 1 km al S, selva baja caducifolia, altitud 930 m, 17 ago. 1982 (flor), G. Lozano Valdez 28 (FCME). Puebla: Acatlán, $18.5 \mathrm{~km}$ al SE, matorral con elementos de selva baja, altitud 1450 m, 2 jul. 1981 (flor), R. Mijares Ballester s.n. (MEXU); Amatitlán, 30 jul. 1942 (fruto), F. Miranda 2168 (MEXU). Morelos: cerros al E de Tlaquiltenango, selva baja decidua, altitud 1100 m, 6 jul. 1941 (flor), F. Miranda 1474 (MEXU).

De lo anterior cabe deducir que esta nueva especie difiere claramente de Nissolia hintonii Sandwith por sus inflorescencias fasciculadas, las hojas de mayor longitud, la pubescencia setoso-glandular escasa, las flores más pequeñas, la forma y el tamaño más pequeño del estandarte, la longitud menor del androceo, del tubo estaminal, de las partes libres de los filamentos del pistilo y del estilo, así como la distribución restringida al bosque tropical caducifolio (o selva baja caducifolia) en altitudes de 650 a $1420 \mathrm{~m}$ (Cuadro 1). Cabe destacar que ambas entidades no son simpátricas, pues mientras $N$. hintonii sólo se conoce de la unidad fisiográfica conocida como Sierra de Taxco, N. ruddiae se presenta únicamente en la parte oriental de la Depresión del Balsas.

El epíteto específico de este nuevo taxon está dedicado a la Dra. Velva E. Rudd (1910-1999), estudiosa de la familia Leguminosae, especialista en el género Nissolia y autora de la revisión del mismo.

\section{AGRADECIMIENTOS}

Se agradece a la M. en C. Martha Martínez Gordillo la diagnosis en latín y la revisión crítica al manuscrito, así como a dos revisores anónimos por las mejoras editoriales sugeridas. 


\section{LITERATURA CITADA}

Miranda, F. y E. Hernández X. 1963. Los tipos de vegetación de México y su clasificación. Bol. Soc. Bot. Méx. 28: 29-179.

Rudd, V. E. 1956. A revision of the genus Nissolia. Contr. U. S. Natl. Herb. 32(2): 173-206.

Rudd, V. E. 1981. Tribe 14. Aeschynomeneae (Benth.) Hutch. (1964). In: Polhill, R. M. \& P. H. Raven. (eds.). Advances in legume systematics. Proc. Internat. Legume Conf. Kew. Part 1: 347-354.

Rzedowski, J. 1978. Vegetación de México. Ed. Limusa. México, D.F. 432 p.p.

Recibido en abril de 2003. Aceptado en junio de 2004. 